\title{
Comparison of the Safety and Efficacy of
} Photoselective Vaporization of the Prostate (PVP) and Transurethral Enucleation with a Bipolar System (TUEB): A Single-Center Retrospective Study

This article was published in the following Dove Press journal: Research and Reports in Urology

Keisuke Kiba'

Yasunori Akashi'

Motokiyo Yoshikawa'

Yutaka Yamamoto (D) ${ }^{\prime}$

Akihide Hirayama (D)

Kiyohide Fujimoto ${ }^{2}$

Hirotsugu Uemura ${ }^{3}$

'Department of Urology, Kindai University Nara Hospital, lkoma-City,

Nara, Japan; ${ }^{2}$ Department of Urology, Nara Medical University, Kashihara-City, Nara, Japan; ${ }^{3}$ Department of Urology, Kindai University Faculty of Medicine, Sayama-City, Osaka, Japan
Correspondence: Keisuke Kiba

Department of Urology, Kindai University Nara Hospital, I248-I Otoda-cho, lkoma-

City, Nara, Japan

Tel +8I-743-77-0880

$\mathrm{Fax}+81-743-77-0890$

Email kiba_uma@yahoo.co.jp
Purpose: The aim of this study was to compare the safety and efficacy of photoselective vaporization of the prostate (PVP) and transurethral enucleation with a bipolar system (TUEB).

Patients and Methods: Patients who underwent PVP or TUEB surgery for lower urinary tract symptoms due to bladder outlet obstruction at our institution from September 2015 to May 2019 were retrospectively reviewed. A total of 83 patients (PVP: $n=45$, TUEB: $n=38$ ) who were available for follow-up at least 12 months after surgery were included. Preoperative characteristics, perioperative parameters, and postoperative outcomes - such as International Prostate Symptom Score (IPSS), quality of life (QoL), maximum urinary flow rate (Qmax), post-void residual urine volume (PVR), and complications-at 3, 6, and 12 months after surgery were compared between the two groups.

Results: Although differences in age, IPSS, and QoL were not significant, a significantly greater prostate volume, lower Qmax, and greater PVR were noted in the TUEB group. In perioperative parameters, a significantly shorter operation time, less change in serum hemoglobin, fewer days of catheterization, and shorter length of stay were observed in the PVP group. As for postoperative outcomes, the IPSS storage subscore and PVR were significantly improved in the TUEB group. As complications, stress urinary incontinence was more frequently observed in the TUEB group, and urethral stricture was more common in the PVP group.

Conclusion: The present data suggest that PVP and TUEB are efficient and safe surgical treatment options. Management of patients undergoing PVP in the perioperative period appears easy. Improvements of subjective and objective parameters were superior after TUEB than after PVP.

Keywords: prostate, prostatic hyperplasia, transurethral resection of prostate, laser therapy

\section{Introduction}

In aging men, benign prostatic hyperplasia $(\mathrm{BPH})$ and related lower urinary tract symptoms (LUTS) are common problems that impact daily activity and quality of life, and may lead to serious outcomes. ${ }^{1}$ Transurethral resection of the prostate (TURP) remains the surgical gold standard for LUTS due to BPH. However, this 
procedure is associated with serious complications, such as intraoperative and postoperative bleeding and hyponatremia. ${ }^{2,3}$

With the recent advances in medical technology, various minimally invasive procedures have replaced TURP. According to the EAU guideline, the choice of surgical technique depends on prostate size, comorbidities of the patient, ability to have anesthesia, patients' preferences, willingness to accept surgery-associated specific sideeffects, availability of the surgical armamentarium, and experience of the surgeon with these surgical techniques. ${ }^{2}$ In accordance with the algorithm for surgical approaches of the guideline, surgical treatment for BPH patients in our hospital includes photoselective vaporization of the prostate (PVP) or transurethral enucleation with a bipolar system (TUEB) in patients with prostate volume $\geq 30 \mathrm{~mL}$.

GreenLight (532-nm laser) PVP, using the oxyhemoglobin molecule as the tissue target chromophobe, has a similar efficacy to TURP, and it is considered a minimally invasive surgical treatment that has a lower risk of bleeding and can be done safely in patients with a large prostate or on oral anticoagulant agents. ${ }^{4,5}$

TUEB is a technique in which the adenoma is dissected and enucleated using a bipolar system. This technique is especially effective for a large prostate, and it is not inferior to open prostatectomy and TURP.,

In recent years, various devices have been developed, and many comparisons between the different techniques have been reported, but there have been no reports of a direct comparison between PVP and TUEB. The aim of this study was to retrospectively compare perioperative parameters, postoperative outcomes, and complications between both techniques for BPH patients in our hospital.

\section{Methods}

Patients who underwent PVP or TUEB surgery for LUTS due to bladder outlet obstruction at our institution from September 2015 to May 2019 were retrospectively reviewed. The 83 patients (PVP: $n=45$, TUEB: $n=38$ ) who were available for follow-up at least 12 months after surgery were included in this study. Exclusion criteria were prostate volume $<30 \mathrm{~mL}$ and loss to follow-up within 12 months.

The International Prostate Symptom Score (IPSS) and quality of life (QOL) score were assessed, and prostate abdominal ultrasonography, uroflowmetry, and urodynamic studies were performed in all patients before surgery. In accordance with BPH severity, surgical treatment was performed in patients with a relative or absolute surgical indication. Each procedure was done with the patient under general or regional anesthesia according to the judgment of the anesthesiologist. Both techniques were performed by five experienced urologists ( 8 years or more, mean 19 years after graduation from university). In accordance with the EAU guidelines, the choice of procedure was left to the discretion of each urologist and patient. After the surgery, an urethral catheter was inserted until the gross hematuria had almost disappeared. After the catheter was removed, the patient was discharged if he could urinate without urinary retention. The questionnaire was completed, uroflowmetry was performed, and complications were assessed at 3,6, and 12 months after surgery.

PVP was performed using a GreenLight 120W HPS system (American Medical System Inc., Minnetonka, MN, USA). A laser fiber was inserted through the working channel of a continuous double-flow 23-Fr resectoscope with normal saline irrigation. The procedure was done according to previously reported techniques, such as the Malek technique, the anterior start technique, and the Basel technique. $^{8}$

TUEB was performed using a bipolar electrosurgical system (UES-40S, Olympus, Tokyo, Japan), a 26-Fr continuous flow resectoscope with a $12^{\circ}$ telescope (Olympus), a specially designed, high-frequency electrode loop with a spatula, and a high-frequency electrode loop (Olympus). The procedure was done according to previously reported techniques. ${ }^{9}$ After enucleation, the prostate adenoma was resected using the standard high-frequency electrode loop, and the prostate chips were evacuated. The removal of adenoma with a tissue morcellator did not conducted.

Patients' characteristics, such as age, prostate volume, IPSS total score, IPSS voiding subscore, IPSS storage subscore, QOL, maximum urine flow rate (Qmax), postvoid residual urine volume (PVR), dutasteride, anticoagulant therapy, the bladder outlet obstruction index (BOOI), and the bladder contractile index (BCI), were compared between the groups. Perioperative parameters, including operation time, change in serum hemoglobin, change in serum sodium, days of catheterization, and length of stay, were compared between the groups. Clinical outcomes were evaluated using the IPSS total score, IPSS voiding subscore, IPSS storage subscore, QOL, Qmax, and PVR at baseline and 3, 6, and 12 months after surgery. Postoperative complications were evaluated using the Clavien-Dindo classification. ${ }^{10}$ Stress urinary incontinence without the need for pharmacological or surgical 
treatment was classified as grade I, and stress urinary incontinence requiring pharmacological treatment was classified as grade II.

Results were compared between the two groups using the Mann-Whitney $U$-test for continuous variables, and the chi-squared test and Fisher's exact test for qualitative variables. Comparison between baseline and each followup visit was performed using the paired $t$-test. A P-value $<0.05$ was considered significant. All statistical analyses were conducted using SPSS ver. 23.

Ethical approval was provided by the Institutional Review Board of Kindai University Nara Hospital as instituted by the Declaration of Helsinki (Number 20-11). The patient consent to review the medical record was not required by the committee due to the retrospective nature of the study. All the data were anonymized and maintained with confidentiality.

\section{Results}

Preoperative characteristics and perioperative data are shown in Table 1. Although the differences in age, IPSS total score, and QoL were not significant, a significantly greater prostate volume (PVP: 59.0 \pm 21.7 , TUEB: 77.7 $\pm 25.3 \mathrm{~mL}$ ), lower Qmax (PVP: 6.9 \pm 3.5 , TUEB: 5.4 $\pm 2.9 \mathrm{~mL} / \mathrm{s}$ ), and greater PVR (PVP: 119 \pm 120 , TUEB: $196 \pm 184 \mathrm{~mL}$ ) were noted in the TUEB group compared with the PVP group. A significantly shorter operation time (PVP: 103.4 \pm 25.5 , TUEB: $122.3 \pm 39.4 \mathrm{~min}$ ), less change in serum hemoglobin (PVP: $1.0 \pm 0.9$, TUEB: $1.5 \pm 0.9 \mathrm{~g} /$ $\mathrm{dL}$ ), fewer days of catheterization (PVP: 2.5 \pm 1.2 , TUEB: $3.2 \pm 1.4$ days), and shorter length of stay (PVP: $6.5 \pm 1.8$, TUEB: $7.7 \pm 1.9$ days) were observed in the PVP group.

Clinical outcomes are shown in Figure 1. In both groups, all parameters were significantly improved from baseline to each follow-up visit. The IPSS storage subscore (PVP: $-4.0 \pm 3.5$, TUEB: $-5.9 \pm 3.5$ ) and PVR (PVP: $-74 \pm 131$, TUEB: $-160 \pm 182 \mathrm{~mL}$ ) at 6 months after surgery were significantly improved in the TUEB group.

Postoperative complications are shown in Table 2. Grade I stress urinary incontinence was more frequently observed in the TUEB group, and urethral stricture was more frequently observed in the PVP group. Re-catheterization was more frequently observed in the PVP group, but there was no significant difference between the groups.

\section{Discussion}

The present data suggest that PVP and TUEB are efficient and safe surgical treatment options.
Although clinical outcomes were basically similar in the two groups, the IPSS storage subscore and PVR at 6 months after surgery were significantly better in the TUEB group. Regarding perioperative parameters, a significantly shorter operation time, less change in serum hemoglobin, fewer days of catheterization, and shorter length of stay were observed in the PVP group. Thus, TUEB may be slightly more effective, and PVP may be a safer procedure with a shorter hospital stay.

Although a direct comparison between PVP and TUEB has not been previously reported, some comparisons between PVP and enucleation procedures other than TUEB have been reported. Regarding objective parameters, Elmansy et al and Sun et al reported a significant improvement in Qmax after HoLEP in a comparison between PVP and HoLEP. ${ }^{11,12}$ The present data showed that, although there was no difference in the improvement in Qmax between the two groups, the improvement in PVR in the TUEB group was superior to that in the PVP group. This supports the fact that enucleation is better than PVP for improving objective parameters. However, Kim et al reported that there were no differences in the improvements of objective parameters in the comparison between PVP and HoLEP with a small prostate volume of less than $40 \mathrm{~mL} .{ }^{13}$ Enucleation of a large prostate results in forming a large cavity, which may therefore contribute to the improvement of objective parameters.

Regarding subjective parameters, the above comparison between PVP and HoLEP reported that there were no significant differences in the improvements in IPSS and QOL. ${ }^{11,13}$ On the other hand, Inyoung et al reported that there were significantly greater improvements in the IPSS total score, IPSS voiding subscore, and IPSS storage subscore in HoLEP than in PVP. Moreover, they reported that the degree of improvement was much greater for voiding symptoms than for storage symptoms due to greater improvement in Qmax in HoLEP. The present data showed that there was no difference in improvement in the IPSS voiding subscore, but there was a significant improvement in the IPSS storage subscore in the TUEB group. Further improvement in Qmax in the TUEB group might have made a difference in improvement in the IPSS voiding subscore.

The above comparison between PVP and HoLEP reported that there were no significant differences in operation time, days of catheterization, and length of stay. The present data showed a significantly shorter operation time, fewer days of catheterization, and shorter 
Table I Preoperative Characteristics and Perioperative Data

\begin{tabular}{|c|c|c|c|c|c|c|c|}
\hline & \multicolumn{3}{|c|}{ PVP $(n=45)$} & \multicolumn{3}{|c|}{ TUEB $(n=38)$} & \multirow[t]{2}{*}{ p value } \\
\hline & Mean & & SD & Mean & & SD & \\
\hline Age $(y)$ & 72.8 & \pm & 6.9 & 72.7 & \pm & 6.3 & 0.596 \\
\hline Prostate volume $(\mathrm{mL})$ & 59.0 & \pm & 21.7 & 77.7 & \pm & 25.3 & $<0.001$ \\
\hline IPSS total score & 19.4 & \pm & 7.6 & 21.6 & \pm & 7.7 & 0.229 \\
\hline Voiding subscore & 11.0 & \pm & 5.4 & 12.1 & \pm & 5.1 & 0.32 \\
\hline Storage subscore & 8.4 & \pm & 3.4 & 9.5 & \pm & 3.8 & 0.181 \\
\hline QOL & 4.6 & \pm & 1.1 & 4.8 & \pm & 1.0 & 0.37 \\
\hline Qmax (mL/s) & 6.9 & \pm & 3.5 & 5.4 & \pm & 2.9 & 0.037 \\
\hline PVR (mL) & 119 & \pm & 120 & 196 & \pm & 184 & 0.03 \\
\hline Urinary retention & \multicolumn{3}{|l|}{$4(9 \%)$} & \multicolumn{3}{|l|}{7 (I8\%) } & 0.33 \\
\hline \multicolumn{8}{|l|}{ Medication } \\
\hline Dutasteride & \multicolumn{3}{|l|}{$23(51 \%)$} & \multicolumn{3}{|l|}{$23(61 \%)$} & 0.39 \\
\hline Anticoagulant agent & \multicolumn{3}{|l|}{$23(51 \%)$} & \multicolumn{3}{|l|}{$6(16 \%)$} & 0.001 \\
\hline Pressure flow study & & & & & & & \\
\hline $\mathrm{BOOI}$ & 73.5 & \pm & 25.0 & 73.2 & \pm & 24.5 & 0.866 \\
\hline $\mathrm{BCl}$ & 112.6 & \pm & 30.3 & 108.6 & \pm & 22.9 & 0.732 \\
\hline Operation time (min) & 103.4 & \pm & 25.5 & 122.3 & \pm & 39.4 & 0.019 \\
\hline Change in serum $\mathrm{Hb}(\mathrm{g} / \mathrm{dL})$ & 1.0 & \pm & 0.9 & 1.5 & \pm & 0.9 & 0.015 \\
\hline Change in serum sodium (mEq/L) & 0.2 & \pm & 2.4 & -0.7 & \pm & 2.4 & 0.209 \\
\hline Length of catheterization (days) & 2.5 & \pm & 1.2 & 3.2 & \pm & 1.4 & 0.016 \\
\hline Length of stay (days) & 6.5 & \pm & 1.8 & 7.7 & \pm & 1.9 & 0.005 \\
\hline
\end{tabular}

Abbreviations: IPSS, International Prostatic Symptom Score; QOL, quality of life; Qmax, peak flow rate; PVR, postvoid residual urine volume; BOOI, bladder outlet obstruction index; $\mathrm{BCl}$, bladder contractility index; PVP, photoselective vaporization of prostate; TUEB, transurethral enucleation with a bipolar system.

length of stay in the PVP group. Including many cases with small prostate volume and the limited operation schedule in the PVP group might have affected operation time. The greater number of cases with a large prostate volume in the TUEB group resulted in a longer time to improve hematuria. This might make a difference in the days of catheterization and the length of stay.

Although the present study did not conduct a comparison limited to cases with a large prostate volume, some studies have reported that TUEB was effective in patients with large prostate volume. A randomized, controlled trial (RCT) of TUEB versus TURP in patients with a prostate volume of $70 \mathrm{~mL}$ or more reported a significantly longer operation time (156 vs $87 \mathrm{~min}$ ) in the TUEB group, but IPSS, QOL score (6.4 vs 11.6, $\mathrm{p}=0.03$, 1.7 vs 2.6, $\mathrm{p}=0.04$, respectively), and Qmax (19.5 vs $15.1 \mathrm{~mL} / \mathrm{s}$, $\mathrm{p}<0.019$ ) at one year after surgery were significantly improved in the TUEB group. ${ }^{6,7}$ There was no significant difference in postoperative complications, and the rate of re-operation at 5 years after surgery was $0 \%$ in the TUEB group and 5\% in the TURP group, respectively. As well as the above comparison between PVP and HoLEP, enucleation was better for improving objective parameters.

In Japan, only one fiber, up to $400,000 \mathrm{~J}$, can be used per PVP surgery. PVP in a large prostate tends to leave residual adenoma. Thus, enucleation may be more suitable in patients with a large prostate in whom the goal is curability. There are several methods of enucleation: plasmakinetic enucleation and laser enucleation with holmium laser, GreenLight laser, diode laser, and thulium laser. ${ }^{14}$ Laser enucleation requires high initial and maintenance costs, whereas TUEB is more economical because it can be performed with a bipolar TURP device. ${ }^{15}$ Thus, TUEB is considered to be an easy enucleation technique to introduce.

With respect to postoperative complications, ClavienDindo Grade I stress urinary incontinence was more frequently observed in the TUEB group in the present study. 


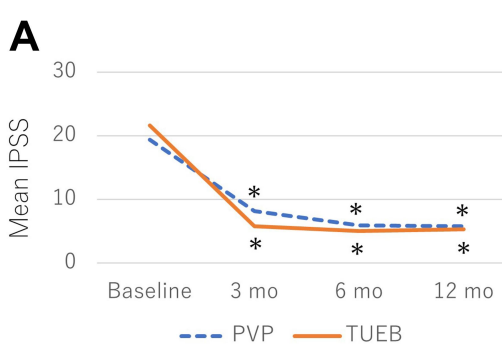

D

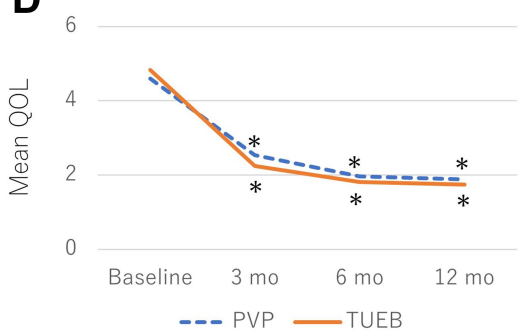

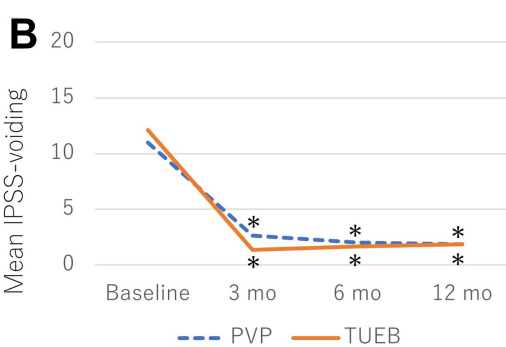

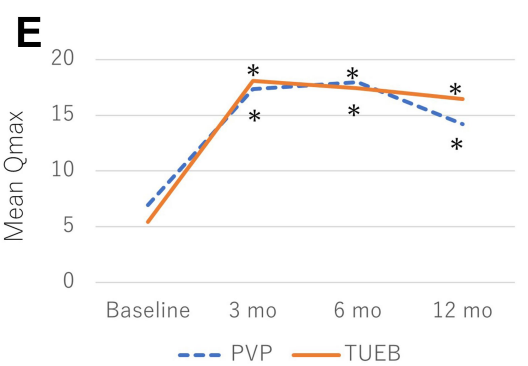



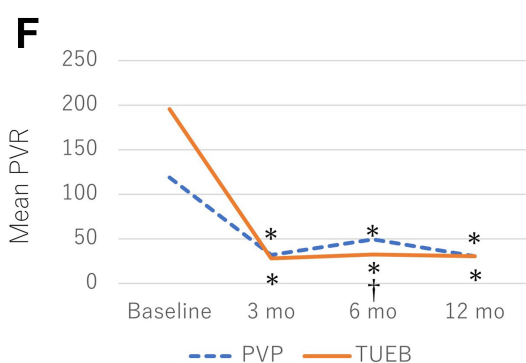

Figure I Mean postoperative values at different points of follow-up are shown for (A) International Prostate Symptom Score (IPSS), (B) IPSS voiding symptom score, (C) IPSS storage symptom score, (D) quality of life (QOL), (E) maximal urine flow rate (Qmax, $\mathrm{mL} / \mathrm{s})$, and (F) postvoid residual urine volume (PVR, mL). *At each follow-up visit, significant differences were found from the value at baseline. ${ }^{\dagger} \mathrm{A}$ significant difference between two groups was found in the change in each parameter from baseline to each follow-up visit.

Abbreviations: PVP, photoselective vaporization of prostate; TUEB, transurethral enucleation with a bipolar system.

A comparison between PVP and HoLEP reported that stress urinary incontinence was more frequently observed in the HoLEP group. ${ }^{12}$ Stress urinary incontinence is considered to be a characteristic complication of enucleation. Stress urinary incontinence is usually caused by the weakness and stretching of the external sphincter due to an enlarged prostate. ${ }^{16}$ On some occasions, enucleation of the adenoma proximal to the external sphincter, especially in the 12o'clock region, may cause transient incontinence. ${ }^{17}$ This

Table 2 Comparison of Postoperative Complications Between PVP and TUEB

\begin{tabular}{|c|c|c|c|c|c|}
\hline \multirow[t]{2}{*}{ Event } & \multicolumn{2}{|c|}{$\begin{array}{l}\text { PVP } \\
(n=45)\end{array}$} & \multicolumn{2}{|c|}{$\begin{array}{l}\text { TUEB } \\
(n=38)\end{array}$} & \multirow[t]{2}{*}{ p value } \\
\hline & $\mathbf{n}$ & $\%$ & $\mathbf{n}$ & $\%$ & \\
\hline Clavien-Dindo Grade I & & & & & \\
\hline Stress urinary incontinence & 0 & 0 & 14 & 37 & $<0.001$ \\
\hline Clavien-Dindo Grade II & & & & & \\
\hline Re-catheterization & 5 & II & 0 & 0 & 0.059 \\
\hline Bleeding & 4 & 9 & I & 3 & 0.369 \\
\hline Urinary tract infection & 2 & 4 & 3 & 8 & 0.656 \\
\hline Stress urinary incontinence & 1 & 2 & 2 & 5 & 0.591 \\
\hline Clavien-Dindo Grade III & & & & & \\
\hline Urethral stricture & 7 & 16 & 0 & 0 & 0.014 \\
\hline Urethral stone & 3 & 7 & 0 & 0 & 0.246 \\
\hline
\end{tabular}

Abbreviations: PVP, photoselective vaporization of prostate; TUEB, transurethral enucleation with a bipolar system. was mostly a short-term complication and resolved effectively after a period of close observation and symptomatic treatment. ${ }^{8}$ In the present study, $37.5 \%$ of patients shortly after surgery in the TUEB group had Grade I stress urinary incontinence, and almost all cases improved after a short period of observation. The incidence of stress urinary incontinence in this study was higher compared to previous reports. The reason for this result may be that we included cases with tiny amounts of incontinence. Urethral stricture was more frequently observed in the PVP group in the present study. Larger scope size was reported to be a risk factor for urethral stricture. ${ }^{18}$ The present data showed that urethral stricture was more frequent in the PVP group, although a smaller scope size was used in the PVP group. However, the reason is unclear, and further study is required. Recatheterization seemed to be more frequently observed in the PVP group, but there was no significant difference between the groups in the present study. Several studies have reported a re-catheterization rate of 2.9-15.3\% following PVP surgery. ${ }^{19-21}$ The present data showed a similar rate of re-catheterization, but all cases improved within a short time. There can be various causes of re-catheterization. Longer catheterization times may be preferred in patients with urinary retention, with very large prostates, the elderly, those on anticoagulants, or those who fail the initial voiding trial due to some swelling/inflammation around the bladder neck or prostatic urethra. Re-catheterization was often seen at the time of PVP introduction at our institution, and the 
incidence has decreased gradually. There may have been a technical problem. The present study showed that there was no significant difference in postoperative bleeding. Laser surgery including PVP can be performed while the patient is taking anticoagulant agents, and it was confirmed that laser surgery could be safely performed in the present study as well. With the increasing number of elderly persons, the number of cases requiring antithrombotic therapy to prevent the onset or recurrence of thrombotic/embolic diseases is increasing, and there are cases in which anticoagulants are difficult to stop. PVP may be suitable for such patients. However, as expected, postoperative bleeding was found in many patients treated with anticoagulant agents. ${ }^{22}$ Careful attention to bleeding complications is necessary in such cases.

This study has several limitations. First, it had a small sample size. Second, there were differences in the operation skills of each surgeon; there were also differences in preoperative prostate volume, Qmax, and PVR, and the choice of procedure was left to the discretion of each urologist. Third, the follow-up period after surgery was relatively short in this study. Fourth, this was a retrospective study. Because each urologist referred to the EAU guidelines, TUEB was often selected in patients with prostate volume greater than $80 \mathrm{~mL}$. Further RCTs are required.

\section{Conclusion}

The present data suggest that PVP and TUEB are efficient and safe surgical treatment options.

TUEB is expected to have slightly better subjective and objective outcomes than PVP. On the other hand, PVP is a minimally invasive treatment that can be performed safely in patients on anticoagulant agents and is expected to shorten hospital stay. It is desirable that urologists familiarize themselves with the characteristics of each surgical procedure and select a surgical procedure that suits the individual patient.

\section{Disclosure}

The authors declare no conflicts of interest.

\section{References}

1. Jacobsen SJ, Girman CJ, Lieber MM. Natural history of benign prostatic hyperplasia. Urology. 2001;58(Suppl 6):5-16. doi:10.1016/ S0090-4295(01)01298-5

2. Oelke M, Bachmann A, Descazeaud A, et al. EAU guidelines on the treatment and follow-up of non-neurogenic male lower urinary tract symptoms including benign prostatic obstruction. Eur Urol. 2013;64 (1):118-140. doi:10.1016/j.eururo.2013.03.004
3. Mayer EK, Kroeze SG, Chopra S, Bottle A, Patel A. Examining the 'gold standard': a comparative critical analysis of three consecutive decades of monopolar Transurethral Resection of the Prostate (TURP) outcomes. BJU Int. 2012;110(11):1595-1601. doi:10.1111/ j.1464-410X.2012.11119.x

4. Bachmann A, Tubaro A, Barber N, et al. 180-W XPS greenlight laser vaporisation versus transurethral resection of the prostate for the treatment of benign prostatic obstruction: 6-month safety and efficacy results of a european multicentre randomised trial-the GOLIATH study. Eur Urol. 2014;65(5):931-942. doi:10.1016/j.eururo.2013.10.040

5. Thomas JA, Tubaro A, Barber N, et al. A multicenter randomized noninferiority trial comparing greenlight-XPS laser vaporization of the prostate and transurethral resection of the prostate for the treatment of benign prostatic obstruction: two-yr outcomes of the GOLIATH study. Eur Urol. 2016;69(1):94-102. doi:10.1016/j. eururo.2015.07.054

6. Kan CF, Tsu HL, Chiu Y, To HC, Sze B, Chan SW. A prospective study comparing bipolar endoscopic enucleation of prostate with bipolar transurethral resection in saline for management of symptomatic benign prostate enlargement larger than $70 \mathrm{G}$ in a matched cohort. Int Urol Nephrol. 2014;46(3):511-517. doi:10.1007/s11255-013-0546-4

7. Zhu L, Chen S, Yang S, et al. Electrosurgical enucleation versus bipolar transurethral resection for prostates larger than $70 \mathrm{ml}$ : a prospective, randomized trial with 5-year followup. $J$ Urol. 2013;189(4):1427-1431. doi:10.1016/j.juro.2012.10.117

8. Gómez Sancha F, Bachmann A, Choi BB, Tabatabaei S, Muir GH. Photoselective vaporization of the prostate (greenLight PV): lessons learnt after 3500 procedures. Prostate Cancer Prostatic Dis. 2007;10 (4):316-322. doi:10.1038/sj.pcan.4500989

9. Liu C, Zheng S, Li H, Xu K. Transurethral enucleation and resection of prostate in patients with benign prostatic hyperplasia by plasma kinetics. J Urol. 2010;184(6):2440-2445. doi:10.1016/j.juro.2010.08.037

10. Dindo D, Demartines N, Clavien PA. Classification of surgical complications: a new proposal with evaluation in a cohort of 6336 patients and results of a survey. Ann Surg. 2004;240(2):205-213. doi:10.1097/01.sla.0000133083.54934.ae

11. Elmansy H, Baazeem A, Kotb A, et al. Holmium laser enucleation versus photoselective vaporization for prostatic adenoma greater than $60 \mathrm{ml}$ : preliminary results of a prospective, randomized clinical trial. J Urol. 2012;188(1):216-221. doi:10.1016/j.juro.2012.02.2576

12. Sun I, Yoo S, Park J, et al. Quality of life after photo-selective vaporization and holmium-laser enucleation of the prostate: 5-year outcomes. Sci Rep. 2019;9(1):8261. doi:10.1038/s41598-019-44686-2

13. Kim KS, Choi JB, Bae WJ, et al. Comparison of photoselective vaporization versus holmium laser enucleation for treatment of benign prostate hyperplasia in a small prostate volume. PLoS One. 2016;11(5):e0156133. doi:10.1371/journal.pone.0156133

14. Dołowy Ł, Krajewski W, Dembowski J, Zdrojowy R, Kołodziej A. The role of lasers in modern urology. Cent European J Urol. 2015;68 (2):175-182. doi:10.5173/ceju.2015.537

15. Chen S, Zhu L, Cai J, et al. Plasmakinetic enucleation of the prostate compared with open prostatectomy for prostates larger than 100 grams: a randomized noninferiority controlled trial with long-term results at 6 years. Eur Urol. 2014;66(2):284-291. doi:10.1016/j. eururo.2014.01.010

16. Kobayashi S, Yano M, Nakayama T, Kitahara S. Predictive risk factors of postoperative urinary incontinence following holmium laser enucleation of the prostate during the initial learning period. Int Braz J Urol. 2016;42(4):740-746.

17. Kuntz RM. Current role of lasers in the treatment of Benign Prostatic Hyperplasia (BPH). Eur Urol. 2006;49(6):961-969. doi:10.1016/j. eururo.2006.03.028

18. Ruszat R, Seitz M, Wyler SF, et al. GreenLight laser vaporization of the prostate: single-center experience and long-term results after 500 procedures. Eur Urol. 2008;54(4):893-901. doi:10.1016/j. eururo.2008.04.053 
19. Capitán C, Blázquez C, Martin MD, Hernández V, de la Peña E, Llorente C. GreenLight HPS 120-W laser vaporization versus transurethral resection of the prostate for the treatment of lower urinary tract symptoms due to benign prostatic hyperplasia: a randomized clinical trial with 2-year follow-up. Eur Urol. 2011;60(4):734-739. doi:10.1016/j.eururo.2011.05.043

20. Lukacs B, Loeffler J, Bruyère $F$, et al. Photoselective vaporization of the prostate with greenlight 120 -w laser compared with monopolar transurethral resection of the prostate: a multicenter randomized controlled trial. Eur Urol. 2012;61(6):1165-1173. doi:10.1016/j. eururo.2012.01.052
21. Horasanli K, Silay MS, Altay B, Tanriverdi O, Sarica K, Miroglu C. Photoselective potassium titanyl phosphate (KTP) laser vaporization versus transurethral resection of the prostate for prostates larger than $70 \mathrm{~mL}$ : a short-term prospective randomized trial. Urology. 2008;71 (2):247-251. doi:10.1016/j.urology.2007.09.017

22. Hori Y, Kuromatsu I, Sugimura Y. Safety and effectiveness of Photoselective Vaporization of the Prostate (PVP) in patients with oral anticoagulant therapy. Hinyokika Kiyo. 2008;54(10):651-656.

\section{Publish your work in this journal}

Research and Reports in Urology is an international, peer-reviewed, open access journal publishing original research, reports, editorials, reviews and commentaries on all aspects of adult and pediatric urology in the clinic and laboratory including the following topics: Pathology, pathophysiology of urological disease; Investigation and treatment of urological disease; Pharmacology of drugs used for the treatment of urological disease. The manuscript management system is completely online and includes a very quick and fair peer-review system, which is all easy to use. Visit http://www.dovepress.com/ testimonials.php to read real quotes from published authors. 